\title{
Fractal Structures in Electrodeposition Process
}

\author{
P. Gębara ${ }^{a, *}$, M. GęBAra ${ }^{b}$ And A. OWCzareK ${ }^{c}$ \\ ${ }^{a}$ Department of Physics, Czestochowa University of Technology, \\ Armii Krajowej 19 Av., 42-200 Częstochowa, Poland \\ ${ }^{b}$ Office of European Projects, Jan Długosz University in Czestochowa, \\ Waszyngtona 4/8 str., 42-200 Częstochowa, Poland \\ ${ }^{c}$ Asten Group Limited, Bór 77/81 str., 42-200 Częstochowa, Poland \\ Doi: 10.12693/APhysPolA.138.287 \\ *e-mail: piotr.gebara@pcz.pl
}

\begin{abstract}
In this work fractal dimension of the dendritic deposits was studied. These dendritic deposits were formed in the electrolysis process at various values of the applied voltage and various molar concentration of the $\mathrm{CuSO}_{4}$ or the $\mathrm{ZnSO}_{4}$ electrolytes. The formation of dendrites was determined by Diffusion Limited Aggregation process occurring during electrolysis of these solutions. Morphology of the obtained deposits was strongly dependent on the molar concentration and voltage applied to the electrodes. In addition, computer simulation was carried out for these deposits using an algorithm based on cellular automata. Theoretical calculations of the fractal dimension value gave the result of 1.7. Experimentally found average values of the fractal dimension were equal either to 1.55 for $\mathrm{CuSO}_{4}$ or to 1.59 for $\mathrm{ZnSO}_{4}$.
\end{abstract}

topics: DLA (Diffusion Limited Aggregation), cellular automata, fractals, dendrites

\section{Introduction}

Aggregation, i.e., the process during which single molecules stick together to form clusters (macroparticles), has been known for a long time. It is commonly found both in nature and in many areas of science and technology, such as engineering materials, polymer chemistry or immunology. Recently, the concepts derived from fractal geometry [1] added a new impetus to researches with respect to this phenomenon. The most interesting structure development is observed in the process of diffusion limited aggregation (DLA). In this process, molecules stick to each other to form the dendritic fractal structure. One of the experiments, as a result of which the fractal dendritic structure can be easily received, is the electrodeposition process [2-4]. This process was used in the presented study to obtain aggregates of copper and zinc, the morphology of which depends mainly on two control parameters: the concentration of the electrolyte, and the potential difference between the electrodes. Under certain conditions (e.g., for either a very thin layer of electrolyte or the electrolyte covered with an additional layer of n-butyl acetate) the aggregation process becomes two-dimensional (2D). Moreover, if the electrolytic deposition is controlled by a single process 0 - diffusion, the metallic deposits exhibit self-similarity, that means they are fractals [2].

\section{Experimental technique and simulation}

The experimental system designed for studying the electrolytic deposition consisted of a cylindrical Petri dish with a diameter of about $50 \mathrm{~mm}$ and a depth of $1.5 \mathrm{~mm}$, filled with a thin layer of an aqueous solution of copper or zinc sulfate; the copper anode in the form of a ring was mounted on the periphery of the vessel, while the graphite cathode was placed in the dish center and touched the electrolyte. The voltage applied to the electrodes was controlled by means of an adjustable power supply. The deposition of metal at the cathode and the sprawling of a cluster was filmed using the Sony DCR 106E and photographed by means of the Nikon Coolpix 3200 camera. The formation of clusters was examined under the voltage values within the range $0-12 \mathrm{~V}$ and various values of the molar concentration of the electrolyte (up to $1 \mathrm{M}$ solution). Figure 1a-c present the scheme of experimental system, the Petri dish, and an image of dendritic structure for copper deposit, respectively.

Mathematical modeling of electrolytic deposition of metallic fractal structures is based on Brownian motion: the metal ions (e.g., $\mathrm{Cu}^{2+}, \mathrm{Zn}^{2+}$ ) move randomly in solution (the movement is described by the random walk model) to be caught by the attracting cathode $[2,4]$. The highest probability of attachment of metal ions occurs in top regions of the dendrites. 


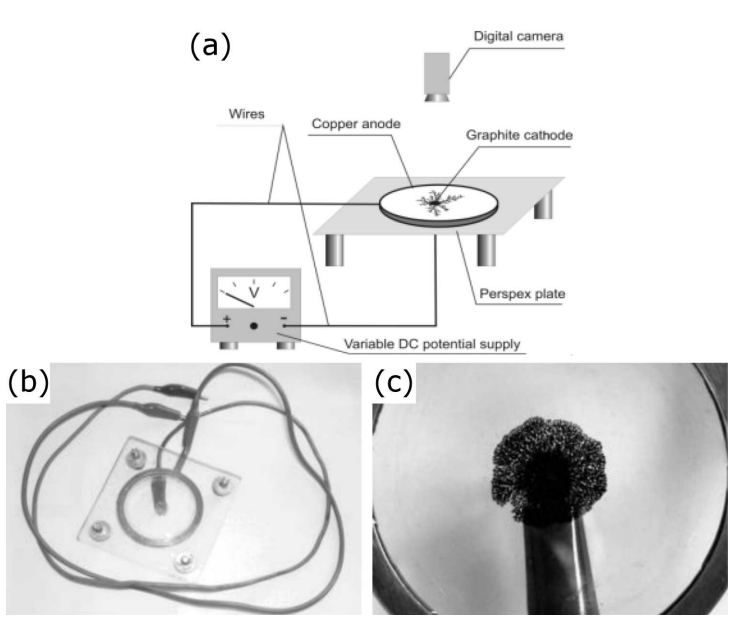

Fig. 1. Scheme of the experimental system (a), Petri dish with connected electrical wires (b), an example of the fractal structure of copper deposit (0.01 M solution of copper sulfate, voltage equal to $4 \mathrm{~V})$.

DLA aggregation type can be simulated using the cellular automata concept and the Mathematica software package developed by Wolfram Research company.

The simulation procedure is as follows [3]:

1. We start with a square array of cells, $n \times n$ in size, all of them of zero value except for the central element of the array, which is given the value of two (it is a nucleus of dendritic crystallization);

2. We establish the process rules: the process will run in a circular region, and the current neighborhood is the von Neumann neighborhood (four possible directions of motion);

3. We place an element of the value of unity (the free particle) on the edge of the array and let it randomly move over the array;

4. There are two scenarios for a free particle: either the particle leaves the area of the array then we forget it and release a new particle at the randomly selected edge point, or the particle stays within the array moving in a chaotic way until it meets the crystallization nucleus — in this case it changes its value from one to two and becomes a part of the immovable nucleus;

5. We place the next free element of the value of unity on the edge of the array at a random location and the process begins again.

\section{Results}

Figure 2 presents the different stages of dendritic crystal growth obtained by computer simulation: black cells represent the molecules forming a crystal, the gray color is used to mark the free particle.

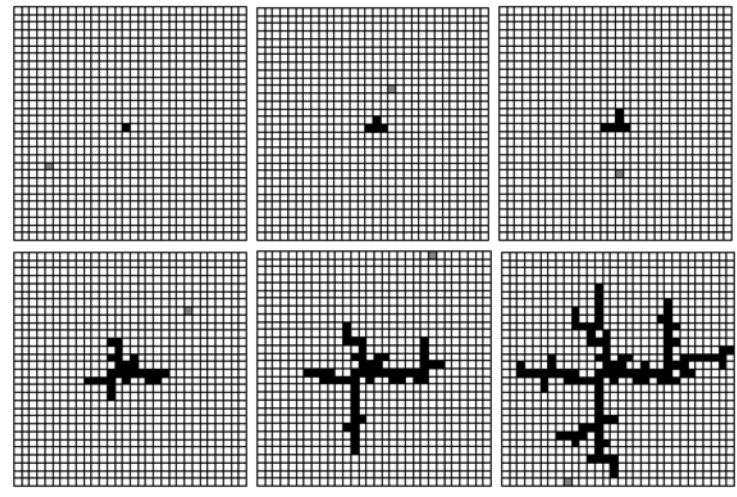

Fig. 2. Simulation of cluster development according to the DLA model for an array of $30 \times 30$ size.

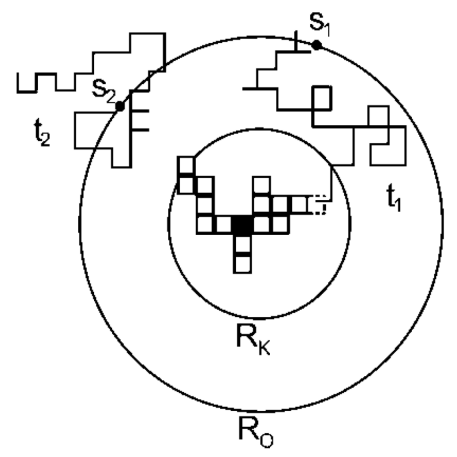

Fig. 3. 2D simulation of the Witten-Sander aggregate [5].

The main problem in this kind of simulation is its duration - the process of formation of dendritic crystals takes a long time for large arrays, so one has to use various tricks to accelerate it. The most time-efficient algorithm is based on the following procedure [5]: the free particle is released at a distance $R_{0}$ (which is a little larger than $R_{k}$ ), the latter being the distance between the center of the crystal cluster and the most distant cell belonging to the cluster (see Fig. 3). If the free particle drifts away farther than $R \approx 3 R_{k}$ (trajectory $\mathrm{t}_{2}$ ), we forget it and release a new free particle at a random point on the circumference of a circle of radius $R_{0}$, which can possibly join the cluster (trajectory $t_{1}$ ).

This approach demands for very careful determination of the appropriate $R_{k}$ value. If the $R_{k}$ value is too small, it is quite likely that the cluster will select the preferential direction of growth during the formation of dendritic crystal, as shown in Fig. 4.

Figure 5 shows the dendritic crystal growth for $R_{k}=5$. Further, we believe that it is the optimum value of $R_{k}$. The $100 \times 100$ array was used for simulation. The time required for generation of such images by means of $1.8 \mathrm{GHz} \mathrm{PC}$ is approximately $15 \mathrm{~min}$.

Fractal dimensions of the generated dendritic structures were calculated in the course of computer simulations. The fractal dimension is defined 

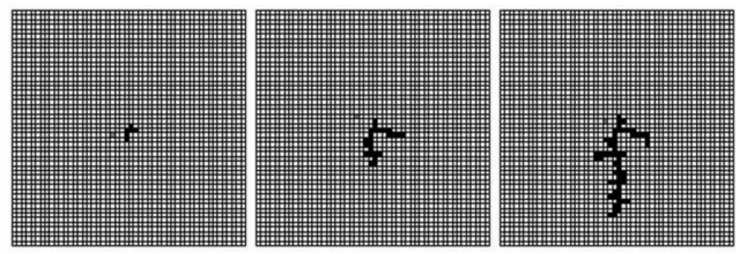

Fig. 4. Computer-simulated image of the DLA aggregate, too small value of $R_{k}\left(R_{k}=2\right)$ resulted in the preferential direction of cluster growth. An array of $50 \times 50$ size was used..

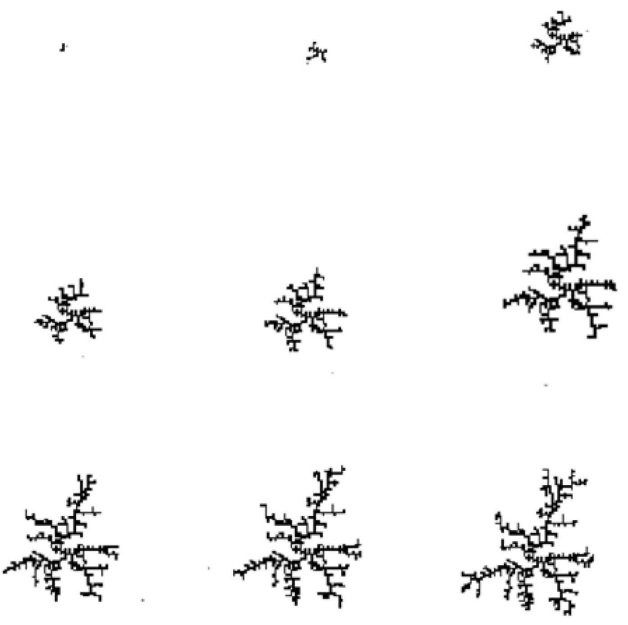

Fig. 5. The subsequent phases of dendritic crystal growth for $R_{k}=5$, the last figure presents the cluster consisting of 700 particles, $100 \times 100$ array.

as $D=\ln (N) / \ln \left(R_{N}\right)$, where $N$ is the number of molecules belonging to the cluster, and $R_{N}$ is the distance from the center of the cluster to its farthest particle. The calculated values oscillated around 1.71 , which is the correct result for the twodimensional aggregates [3].

Figure 6 illustrates the experimental formation of the metallic zinc during electrolysis, the aggregate taking the form of a few dendrites. Then a series of photographs shown in Fig. 7 presents the formation of dendritic fractal structure from copper atoms.

The current flowing through the electrolyte and the length of the longest aggregate were measured in order to determine the fractal dimension of electrolytic deposits. According to the first Faraday's law, the mass $m$ of the product deposited at the electrode is proportional to the total electric charge $q$ passed through the electrolyte, which in turn can be calculated from the value of the current $I$ flowing in the time $t$ :

$$
\begin{aligned}
& m=k q \\
& q=I t
\end{aligned}
$$

where $I$ denotes the current and $t$ is the flowing time.
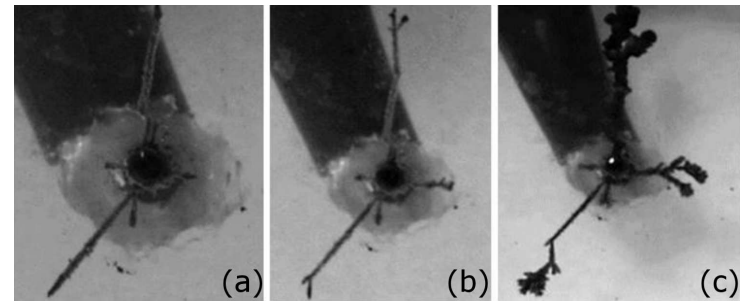

Fig. 6. Experimentally observed zinc aggregation process: (a) the growth time $t=90 \mathrm{~s}$, (b) $t=150 \mathrm{~s}$, (c) $t=270 \mathrm{~s}$. Molar concentration of $\mathrm{ZnSO}_{4}: 1 \mathrm{M}$, the voltage between the electrodes $8 \mathrm{~V}$.

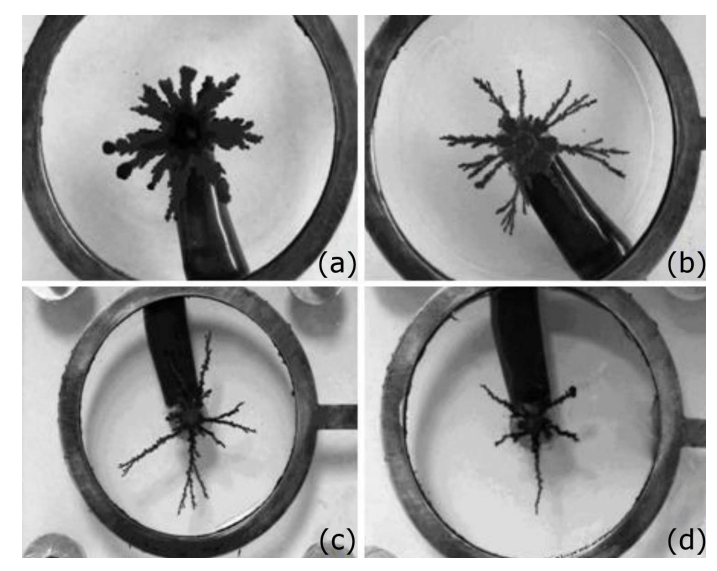

Fig. 7. Photographs of the copper deposit structure for various molar concentrations of $\mathrm{CuSO}_{4}$ : (a) $0.1 \mathrm{M}$, the growth time $t=780 \mathrm{~s}$, (b) $0.3 \mathrm{M}$, $t=1200 \mathrm{~s}$, (c) $0.5 \mathrm{M}, t=1050 \mathrm{~s}$, (d) $1 \mathrm{M}, t=480 \mathrm{~s}$. The applied voltage: $10 \mathrm{~V}$.

Therefore:

$$
m=k I t
$$

where $k$ stands for the electrochemical equivalent of an element. Taking into account that mass is strongly related to radius of deposition, according to following relation [1]:

$$
m \propto R^{D}
$$

where $m$ is the mass of the deposit, $R$ denotes the length of the aggregate radius, and $D$ is the fractal dimension. The number of deposited particles is given by [1]:

$$
N=C R^{D},
$$

where $N$ is the number of particles in the aggregate, and $C$ is the proportionality constant.

In addition, the number of particles caught in unit time is given by [1]:

$$
\Delta N=\frac{I}{q} \Delta t
$$

where $I$ denotes the current, and $q=3.210^{-19} \mathrm{C}$ is the electric charge of a single ion.

By converting both sides of (5) to the logarithmic form, we obtain:

$$
\log (N)=D \log (R)+\log (C),
$$


Number of particles and the radius of the aggregate are known, therefore using linear regression we can determine the fractal dimension $D$. The specified fractal dimension averaged 1.55 and 1.59 for copper and zinc deposits, respectively.

\section{Conclusions}

The conducted experiments offer an interesting and accessible way to the understanding of such phenomena as aggregation, electrolysis, randomness in physical systems and Brownian motion. Additionally, the examination of structures of the obtained deposits and of their morphological changes allow for better understanding of the processes of metal deposition on the cathodes of the various types of batteries [1]. Such deposition is very harmful and significantly shortens battery life. Experimentally found values of the fractal dimension correspond quite well to the theoretical value. The morphology of the obtained deposits depends on the electrolyte molar concentration and the voltage applied to electrodes. The densely packed structure or radial ordering was obtained for low molar concentrations and low voltage values. The dendritic structure occurred for the voltage range 6-12 V and the electrolyte concentration of 0.1 to $1 \mathrm{M}$.

\section{Acknowledgments}

This work was supported by the Rector of Częstochowa University of Technology, prof. Norbert Sczygiol.

\section{References}

[1] H.O. Peitgen, H. Jürgens, D. Saupe, Chaos, Fractals, Springer, New York (1992).

[2] M. Matsushita, Experimental Observation of Aggregations in: The Fractal Approach To Heterogeneous Chemistry, John Wiley \& Sons (1989), p. 161.

[3] T.A. Witten, L.M. Sander, Phys. Rev. Lett. 47, 1400 (1981).

[4] R. Brady, R. Ball, Nature 309, 225 (1984).

[5] P. Maekin, Computer Simulation of Growth and Aggregation Processes in: On Growth And Form. Fractal And Non-Fractal Patterns In Physics, Eds. H.E. Stanley, N. Ostrowsky, NATO ASI Series, 1986, p. 111. 